\title{
Manipulating Multi-qudit Entanglement Witnesses by Using Linear Programming
}

\author{
M. A. Jafarizadeh ${ }^{a, b, c *}$, G. Najarbashi ${ }^{a, b \dagger}$, H. Habibian ${ }^{a} \ddagger$ \\ ${ }^{a}$ Department of Theoretical Physics and Astrophysics, Tabriz University, Tabriz 51664, Iran. \\ ${ }^{b}$ Institute for Studies in Theoretical Physics and Mathematics, Tehran 19395-1795, Iran. \\ ${ }^{c}$ Research Institute for Fundamental Sciences, Tabriz 51664, Iran.
}

August 13, 2018

*E-mail:jafarizadeh@tabrizu.ac.ir

${ }^{\dagger}$ E-mail:najarbashi@tabrizu.ac.ir

${ }^{\ddagger}$ E-mail:hesam-habibian@sicatechdec.com 


\begin{abstract}
A new class of entanglement witnesses (EWs) called reduction type entanglement witnesses is introduced, which can detect some multi-qudit entangeled states including PPT ones with Hilbert space of dimension $d_{1} \otimes d_{2} \otimes \ldots \otimes d_{n}$. The novelty of this work comes from the fact that the feasible regions turn out to be convex polygons, hence the manipulation of these EWs reduces to linear programming which can be solved exactly by using simplex method. The decomposability and non-decomposability of these EWs are studied and it is shown that it has a close connection with eigenvalues and optimality of EWs. Also using the Jamiołkowski isomorphism, the corresponding possible positive maps, including the generalized reduction maps of Ref. [21], are obtained.

Keywords: Entanglement Witness, Multi-qudit, Optimality, Linear Programming, Feasible Region .

PACs Index: 03.65.Ud
\end{abstract}




\section{Introduction}

Quantum entangled states lie at the heart of the rapidly developing field of quantum information science, which encompasses important potential applications such as quantum communication, quantum computation [1, 2, 3]. However, the fundamental nature of entangled states has tantalized physicists since the earliest days of quantum mechanics, and even today is by no means fully understood. One of the most basic problems is that how can one tell if a quantum state is entangled?, and how entangled is it still after some noisy quantum process.

Here we will deal with the states of finite dimensional Hilbert space $\mathcal{H}=\mathcal{H}_{d_{1}} \otimes \ldots \otimes \mathcal{H}_{d_{n}}$. A density matrix $\rho$ is called unentangled or separable if it can be written as a convex combination of pure product states as $|\gamma\rangle=\left|\alpha^{(1)}\right\rangle \otimes \ldots \otimes\left|\alpha^{(n)}\right\rangle$. If no such convex linear combination exists for a given $\rho$, then the state is called entangled. Although, in the case of pure states of bipartite systems it is to check whether a given state is, or is not entangled, the question is yet an open problem in the case of mixed states (see the recent good reviews [4, 5, 6, 7]). There have been many efforts in recent years to analyze the separability and quantitative character of quantum entanglement. The Bell inequalities satisfied by a separable system give the first necessary condition for separability [8]. In 1996, Peres made an important step towards proving that, for a separable state, the partial transposition with respect to one subsystem of a bipartite density matrix is positive, $\rho^{T_{A}} \geq 0$ [9]. By establishing a close connection between positive map theory and separability, Horodecki et al. promptly showed that this is sufficient condition for separability for bipartite systems of $2 \otimes 2$ and $2 \otimes 3$ [10]. Regarding the quantitative character of entanglement, Wootters succeeded in giving an elegant formula to compute the entanglement of formation [11] of $2 \otimes 2$ mixtures, thus giving also a separability criterion [12]. An alternative method to detect entanglement is to construct so-called entanglement witnesses [10, 13, 14, 15] and positive maps [16]. Entanglement witnesses are physical observables that can detect the presence of entanglement. Recently many attempts have been made to use the 
convex optimization methods as a robust tools in most quantum information areas including construction of EWs [17, 18, 19, 14, 15].

The main motivation for the present investigation is the remarkable fact that there is no evidence that when and where the manipulation of the EWs reduces to LP which can be solved exactly. For example, the first author and his collaborators have found certain examples of Bell diagonal EWs and generalized $d \times d$ Choi entanglement witnesses [14, 15], by approximate LP method. Indeed in most cases determining feasible regions needs to use numerical calculation and consequently the problem is solved approximately. Therefore to figure out the problems which can be easily solved exactly in a optimal way and also can be generalized to an arbitrary number of particles with different Hilbert spaces is the main purpose of this paper. To this aim we introduce a new class of $\left(d_{1} \otimes d_{2} \otimes \ldots \otimes d_{n}\right)$-multi-qudit EWs called reduction type entanglement witnesses(REWs) and show that the computational difficulty in solving such problems reduces to LP which can be solved by simplex method[20]. In these cases the feasible regions are simplexes and the minimum value of optimization problem is achieved on their apexes. On the other hand we show that the EWs corresponding to hyperplanes surrounding feasible regions are optimal. Another advantage of this work is the fact that all REWs can be written in terms of some positive operators and optimal EWs. Also in most cases the decomposability of REWs is rather determined, where two of their eigenvalues plays an important role in this issue. Another important consequences of such EWs are the positive maps including the generalized reduction map [21], which can be obtained from these EWs or their tensor product, via Jamiołkowski isomorphism. Finally a class of entangled density matrix including PPTES are provided which can be detected by such REWs and consequently they determine the non-decomposability of REWs. At the end we carry out in detail some particular cases such as multi-qubit and $2 \otimes 3 \otimes 4$ and generalized reduction EW. The paper organized as follows: In section 2 we give a brief review of EWs. In section 3 we explain the general scheme of linear programming. In section 4, we introduce a new class of 
EWs which can be put in the realm of LP, since their feasible regions are convex polygons (indeed simplexes) which can be exactly determined. In section 5, we show that all EWs corresponding to hyperplanes surrounding the feasible regions are optimal. Section 6 is devoted to some interesting examples such as: multi-qubit REWs, $(d \otimes d \ldots \otimes d)$ multi-qudit REWs and $2 \otimes 3 \otimes 4$ REW. Section 7 deals with two examples of LP type, where the first one can be solved exactly by the prescription of this paper. In section 8 we introduce some entangled and PPT states which can be detected by REWs and the decomposability of REWs is discussed. In section 9 by using Jamiołkowski isomorphism, the relation between REWs and positive maps is explained. The paper is ended with a brief conclusion and three appendices.

\section{Entanglement witness}

As mentioned above in the introduction, one of the pragmatic approach to detect entanglement is to construct entanglement witnesses. Let us briefly recall what these operators are.

Definition 1. A Hermitian operator $\mathcal{W}$ is called an entanglement witness detecting the entangled state $\rho_{e}$ if $\operatorname{Tr}\left(\mathcal{W} \rho_{e}\right)<0$ and $\operatorname{Tr}\left(\mathcal{W} \rho_{s}\right) \geq 0$ for all separable state $\rho_{s}$.

So, if we have a state $\rho$ and we measure $\operatorname{Tr}(\mathcal{W} \rho)<0$, we can be sure that $\rho$ is entangled. This definition has a clear geometrical meaning. The expectation value of an observable depends linearly on the state. Thus, the set of states where $\operatorname{Tr}(\mathcal{W} \rho)=0$ holds is a hyperplane in the set of all states, cutting this set into two parts. In the part with $\operatorname{Tr}(\mathcal{W} \rho)>0$ lies the set of all separable states, the other part ( with $\operatorname{Tr}(\mathcal{W} \rho)<0$ ) is the set of state detected by $\mathcal{W}$. From this geometrical interpretation it follows that all entangled states can be detected by witness. Indeed for each entangled state $\rho_{e}$ there exist an entanglement witness detecting it [10].

Definition 2. An $E W$ is decomposable (d-EW) iff there exists operators $\mathcal{P}, \mathcal{Q}_{i}$ with

$$
\mathcal{W}=\mathcal{P}+\mathcal{Q}_{1}^{T_{A}}+\mathcal{Q}_{2}^{T_{B}}+\ldots+\mathcal{Q}_{N}^{T_{Z}} \quad \mathcal{P}, \mathcal{Q}_{i} \geq 0
$$


where partial transpose taken with respect to some subsystems and it is non-decomposable if it can not be put in this form [19].

Only non-decomposable EWs can detect PPT entangled states that is those density matrices which have positive partial transposition with respect to each subsystems [22].

\section{Manipulating EWs by LP method}

This section deals with basic definitions of linear programming(LP) and general scheme to construct EWs by an exact LP method.

To this aim first we consider a Hermitian operator $\mathcal{W}$ with some negative eigenvalues

$$
\mathcal{W}=\sum_{i} a_{i} \sigma_{i}
$$

where $\sigma_{i}$ are positive operators, with $0 \leq \operatorname{Tr}\left(\sigma_{i} \rho_{s}\right) \leq 1$ for every separable states $\rho_{s}$. Note that, the condition $0 \leq \operatorname{Tr}\left(\sigma_{i} \rho_{s}\right) \leq 1$ is not always required. It is used here only to simplify analyzing the problem and pave the way to generalize the prescription to multi-qudits with arbitrary higher dimensions as will be discussed in the following.

The minimum value of $\mathcal{F}=\operatorname{Tr}\left(\mathcal{W} \rho_{s}\right)$ achieves for pure product state, since every mixed $\rho_{s}$ can be written as a convex combination of pure product states (due to the convexity of separable region) as $\rho_{s}=\sum_{i} p_{i}\left|\gamma_{i}\right\rangle\left\langle\gamma_{i}\right|$ with $p_{i} \geq 0$ and $\sum_{i} p_{i}=1$, hence we have

$$
\operatorname{Tr}\left(\rho_{s} \mathcal{W}\right)=\sum_{i} p_{i} \operatorname{Tr}\left(\mathcal{W}\left|\gamma_{i}\right\rangle\left\langle\gamma_{i}\right|\right) \geq C_{\min }
$$

$$
\text { with } \quad C_{\text {min }}=\min \mathcal{F}:=\operatorname{Tr}(\mathcal{W}|\gamma\rangle\langle\gamma|) \quad|\gamma\rangle \in D_{\text {prod }},
$$

where $D_{\text {prod }}$ denotes the set of product states. Thus we need to find the pure product state $\left|\gamma_{\text {min }}\right\rangle$ which minimize $\operatorname{Tr}(\mathcal{W}|\gamma\rangle\langle\gamma|)$. Now, as the pure product state $|\gamma\rangle$ varies, the map defined by $P_{i}=\operatorname{Tr}\left(\sigma_{i}|\gamma\rangle\langle\gamma|\right)$ maps the set $D_{\text {prod }}$ into a region inside the hypercube defined by $0 \leq P_{i} \leq 1 \quad i=1,2, \ldots, N$. This is not all circumstances. An important and difficult task 
is to find the convex region (called feasible region) inside this hypercube which comes from $\mathcal{F}_{\mathcal{W}}:=\operatorname{Tr}\left(\mathcal{W} \rho_{s}\right)$ as $\rho_{s}$ varies on $D_{\text {sep }}$, where $D_{\text {sep }}$ denotes the set of separable states. Here in this work we are interested in the EWs with the feasible regions of simplexes (or at most convex polygons)types, such that the manipulating these EWs amounts to

$$
\begin{aligned}
\operatorname{minimize} & \mathcal{F}_{\mathcal{W}}:=\operatorname{Tr}\left(\mathcal{W} \rho_{s}\right)=\sum_{i} a_{i} P_{i} \\
\text { subject to } & \sum_{i=1}^{N}\left(c_{i j} P_{i}-d_{i}\right) \geq 0 \quad j=1,2, \ldots
\end{aligned}
$$

where $c_{i j}, d_{i} i, j=1,2, \ldots$ are parameters of hyper-planes surrounding the feasible regions. Therefore, the corresponding boundary points of feasible region will minimize exactly $\mathcal{F}_{w}$, thus the required task reduces to LP problem which can be solved by the well-known simplex method [20].

\section{$4 \quad\left(d_{1} \otimes d_{2} \otimes \ldots \otimes d_{n}\right)$ multi-qudit reduction type EWs}

In this section we consider $n$ Particles with arbitrary dimensions. Without loss of generality particles can be arranged so that $d_{1} \leq d_{2} \leq \ldots \leq d_{n}$. The discussion of some special cases is postponed to Section 6. We introduce and parameterize the multi-qudit reduction-type entangled witnesses (REWs) labeled by subscript $\mathrm{R}$ as

$$
\mathcal{W}_{R}^{(n)}=\sum_{S \varsubsetneqq N^{\prime}} b_{S} \sigma_{S}+d_{1} b_{2, \ldots, n}\left|\psi_{00 \ldots 0}\right\rangle\left\langle\psi_{00 \ldots 0}\right|+\sum_{S \varsubsetneqq N^{\prime}} a_{S}^{\prime} \sigma_{S}^{\prime},
$$

where $N^{\prime}=\{2, \ldots, n\}$, with $b_{\emptyset}=b_{1}, a_{\emptyset}^{\prime}=a_{1}^{\prime}$ and $\sigma_{S}, \sigma_{S}^{\prime}$ defined as

$$
\sigma_{S}=\sum_{i=0}^{d_{1}-1}|i\rangle\langle i| \otimes O_{i}^{(2)} \otimes \ldots \otimes O_{i}^{(n)} \quad \text { with } \quad O_{i}^{(k)}=\left\{\begin{array}{ccc}
|i\rangle\langle i| & \text { if } & k \in S \\
I & \text { if } & k \notin S
\end{array}\right.
$$

and

$$
\sigma_{S}^{\prime}=\sum_{i_{1}=0}^{d_{1}-1} \sum_{i_{2}=0}^{d_{2}-1} \ldots \sum_{i_{n}=0}^{d_{n}-1}\left|i_{1}\right\rangle\left\langle i_{1}\right| \otimes O_{i_{2}}^{(2)} \otimes \ldots \otimes O_{i_{n}}^{\prime(n)}
$$


with

$$
O_{i_{k}}^{(k)}= \begin{cases}\left|i_{1}\right\rangle\left\langle i_{1}\right| \delta_{i_{1} i_{k}} & \text { if } k \in S \\ \left|i_{k}\right\rangle\left\langle i_{k}\right| \prod_{k \neq m=1}^{n}\left(1-\delta_{i_{k} i_{m}}\right) & \text { if } k \notin S, \quad|S| \leq n-3 \quad \text { and } i_{2}, \ldots, i_{n} \leq d_{1}-1 \\ 0 & \text { if } k \notin S, \quad|S|=n-2 \quad \text { and } i_{2}, \ldots, i_{n} \leq d_{1}-1 \\ \left|i_{k}\right\rangle\left\langle i_{k}\right|\left(1-\delta_{i_{k} i_{1}}\right) & \text { otherwise }\end{cases}
$$

, respectively and

$$
\left|\psi_{00 \ldots 0}\right\rangle:=\frac{1}{\sqrt{d_{1}}} \sum_{i=0}^{d_{1}-1}\left|i^{(1)}\right\rangle\left|i^{(2)}\right\rangle \ldots\left|i^{(n)}\right\rangle
$$

is maximally entangled state. In this notation we have $\sigma_{\emptyset}^{\prime}=\sigma_{1}^{\prime}$. Obviously for multi-qubit system none of $\sigma_{S}^{\prime}$ exists. The number of $P_{S}^{\prime}$ depends on the dimensions of particles $d_{i}$ 's and it can take one of the following values

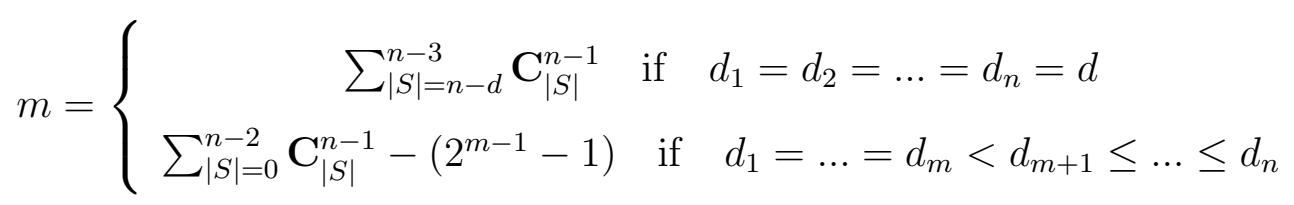

We introduce the new more convenient parameters $a_{1}=b_{1}=b_{\emptyset}, a_{S}=b_{S}+\sum_{S^{\prime} \varsubsetneqq S} b_{S^{\prime}}$, instead of $b_{S}$ 's. In order to turn the observable (4.5) to an EW, we need to choose its parameters in such a way that it becomes a non-positive operator with positive expectation values in any pure product state $|\gamma\rangle=\left|\alpha^{(1)}\right\rangle \otimes \ldots \otimes\left|\alpha^{(n)}\right\rangle$.

Now it is the time to reduce the problem to LP one. Io order to determine the feasible region, we need to know the apexes, namely the extremum points, to construct the hyperplanes surrounding the feasible regions. Suppose that $|\gamma\rangle$ be a pure product state with $\left|\alpha^{(k)}\right\rangle=$ $\left(\alpha_{0}^{(k)}, \alpha_{1}^{(k)}, \ldots, \alpha_{d_{k}-1}^{(k)}\right)^{T}$ and let

$$
\begin{gathered}
P_{S}:=\operatorname{Tr}\left(\sigma_{S}|\gamma\rangle\langle\gamma|\right)=\sum_{i=0}^{d_{1}-1}\left|\beta_{i}^{(1)} \beta_{i}^{(2)} \ldots \beta_{i}^{(n)}\right|^{2}, \quad \beta_{i}^{(k)}=\left\{\begin{array}{ccc}
\alpha_{i}^{(k)} & \text { if } & k \in\{1\} \cup S \\
1 & \text { if } \quad k \notin\{1\} \cup S
\end{array}\right. \\
P_{2, \ldots, n}:=d_{1}\left|\left\langle\psi_{00 \ldots 0} \mid \gamma\right\rangle\right|^{2}=\left|\sum_{i=0}^{d_{1}-1} \alpha_{i}^{(1)} \alpha_{i}^{(2)} \ldots \alpha_{i}^{(n)}\right|^{2} \\
P_{S}^{\prime}:=\operatorname{Tr}\left(\sigma_{S}^{\prime}|\gamma\rangle\langle\gamma|\right),
\end{gathered}
$$


which all lie in the interval $[0,1]$ (see appendix I). The number of $P_{S}$ is $2^{n-1}-1$ (cardinality of the power set of $N^{\prime}$ excepted $\left.\{\emptyset\}\right)$.

The extremum points or apexes consist of

a. origin: $\quad P_{S}=0, \quad P_{S}^{\prime}=0 \forall S \subseteq N^{\prime} \quad$ which corresponds to the following choice of pure product state

$$
\begin{gathered}
\left|\alpha^{(1)}\right\rangle=\left(\begin{array}{lllll}
1 & 0 & 0 & \ldots & 0
\end{array}\right)^{T} \\
\left|\alpha^{(k)}\right\rangle=\left(\begin{array}{lllll}
0 & 1 & 0 & \ldots & 0
\end{array}\right)^{T} \quad k \in N^{\prime}
\end{gathered}
$$

b. $\quad P_{S}=1, \quad P_{S^{\prime}}=1 \forall S^{\prime} \subseteq S, \quad P_{N^{\prime}}=0, \quad P_{S^{\prime \prime}}=0 \forall S^{\prime \prime} \subseteq N^{\prime} \backslash S, \quad P_{S}^{\prime}=0 \forall S \subseteq N^{\prime}$ which can be reached by choosing the following pure product states

$$
\begin{aligned}
& \left|\alpha^{(k)}\right\rangle=\left(\begin{array}{lllll}
1 & 0 & 0 & \ldots & 0
\end{array}\right)^{T} \quad k \in\{1\} \cup S, \\
& \left|\alpha^{(k)}\right\rangle=\left(\begin{array}{llllll}
0 & 1 & 0 & \ldots & 0
\end{array}\right)^{T} \quad k \notin\{1\} \cup S .
\end{aligned}
$$

Obviously if $P_{S}=1$, then for all $S^{\prime} \subseteq S, P_{S^{\prime}}=1$, thus for $S=N^{\prime}$, we get the following important apex

c. $\quad P_{S}=1, P_{S}^{\prime}=0 \forall S \subseteq N^{\prime}$.

which can be obtained by choosing

$$
\left|\alpha^{(k)}\right\rangle=\left(\begin{array}{lllll}
1 & 0 & 0 & \ldots & 0
\end{array}\right)^{T} \quad \forall k \in\{1\} \cup N^{\prime}
$$

d. $P_{S}^{\prime}=1, P_{S^{\prime}}=1 \forall S^{\prime} \subseteq S \subseteq N^{\prime}$, the others are zero.

The last category arise from pure product state with the components of the form $\alpha_{0}^{(1)}=\alpha_{0}^{(k)}=$ 1 , if $k \in S$ and $\alpha_{k-1}^{(k)}=1$, if $k \notin S$, i.e.,

$$
\begin{aligned}
& \left|\alpha^{(k)}\right\rangle=\left(\begin{array}{lllll}
1 & 0 & 0 & \ldots & 0
\end{array}\right)^{T} \quad k \in\{1\} \cup S,
\end{aligned}
$$

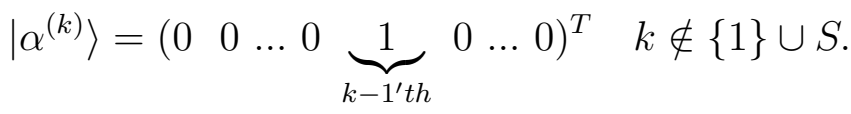


Regarding the above consideration, we are now ready to state the feasible region.

To this aim we first prove that, $2^{n-1}+m$ extremum points obtained above form the apexes of $\left(2^{n-1}+m\right)$-simplex in Euclidean space of dimension $N=2^{n-1}+m-1$. To this purpose we consider the convex hull of theses points, i.e. draw $N+1$ hyperplanes passing through each combination of $N$ points out of $N+1$ ones $\left(\mathbf{C}_{N}^{N+1}=N+1\right)$. Now, we get a bounded region formed from their intersection which is the required feasible region of $\mathcal{W}_{R}^{(n)}$ and it is obviously a $\left(2^{n-1}+m\right)$ - simplex. It is strait forward to show that the feasible region can be obtained by taking the expectation values of

$$
{ }^{(S)} \mathcal{W}_{\text {opt }}^{(n)}=a_{S}\left(\sigma_{S}+\sum_{S \subsetneq S^{\prime} \neq N^{\prime}}(-1)^{|S|+\left|S^{\prime}\right|} \sigma_{S^{\prime}}+d_{1}(-1)^{|S|+\left|N^{\prime}\right|}\left|\psi_{00 \ldots 0}\right\rangle\left\langle\psi_{00 \ldots 0}\right|-\sigma_{S}^{\prime}\right) \quad S \varsubsetneqq N^{\prime}
$$

in pure product states (see appendix II). Now, in order that $\mathcal{W}_{R}^{(n)}$ to be an EW, the following expectation values

$$
\mathcal{F}\left(P_{2}, P_{3}, \ldots, P_{1}^{\prime}, P_{2}^{\prime}, \ldots\right):=\operatorname{Tr}\left(\mathcal{W}_{R}^{(n)}|\gamma\rangle\langle\gamma|\right)=\sum_{S \subsetneq N^{\prime}} b_{S} P_{S}+b_{2, \ldots, n} P_{2, \ldots, n}+\sum_{S \varsubsetneqq N^{\prime}} a_{S}^{\prime} P_{S}^{\prime}
$$

must be positive. So our task is to solve the following LP problem

$$
\begin{aligned}
\text { minimize } & \sum_{S \subsetneq N^{\prime}} b_{S} P_{S}+b_{2, \ldots, n} P_{2, \ldots, n}+\sum_{S \subsetneq N^{\prime}} a_{S}^{\prime} P_{S}^{\prime} \\
\text { subject to } & \left\{\begin{array}{l}
P_{S}-P_{S}^{\prime}+\sum_{S \subsetneq S^{\prime}}(-1)^{|S|+\left|S^{\prime}\right|} P_{S^{\prime}} \geq 0 \\
\forall P_{S}^{\prime} \geq 0
\end{array}\right.
\end{aligned}
$$

Putting the coordinates of apexes in Eq.(4.10) yields all $a_{S} \geq 0$ and $a_{S}+a_{S}^{\prime} \geq 0$. As we stated in previous section, all $P_{S}$ and $P_{S}^{\prime}$ lie in the closed interval $[0,1]$. Now, $\mathcal{F}$ is a linear function of $P_{S}$ and $P_{S}^{\prime}$ and if we require it to be positive on the apexes (which are extremum points), then it will be positive in the whole feasible region.

At the end we need to know all eigenvalues of $\mathcal{W}_{R}^{(n)}$ which consist of $a_{S}, a_{S}+a_{S}^{\prime}, \omega_{1}=a_{N^{\prime}}-$ $b_{N^{\prime}}$ and $\omega_{2}=d_{1} a_{N^{\prime}}-\left(d_{1}-1\right) \omega_{1}$. Since $a_{S}, a_{S}+a_{S}^{\prime} \geq 0$, then one of the remaining eigenvalues: $\omega_{1}$ and $\omega_{2}$ must be negative to guarantee $\mathcal{W}_{R}^{(n)}$ to be an EW. 


\section{$5 \quad$ Optimality of ${ }^{(S)} \mathcal{W}_{\text {opt }}^{(n)}$}

After determining the feasible regions, one needs to know whether the boundary of EWs is formed by optimal EWS. An EW is optimal if a positive operator $P$ is subtracted from that then it will be no longer an EW [13.

Note that the EWs corresponding to hyperplanes surrounding feasible regions of $\mathcal{W}_{R}^{(n)}$ are optimal since they cover the simplex feasible region in an optimal way (see appendix III). Thus, the structure of the optimal EWs ${ }^{(S)} \mathcal{W}_{\text {opt }}^{(n)}$ characterizes the boundary of REWs $\mathcal{W}_{R}^{(n)}$. In fact, from the results of this Section it will become clear that we can restrict ourselves to the structure of the optimal EWs corresponding to hyperplanes surrounding feasible regions. In other words optimal EWs ${ }^{(S)} \mathcal{W}_{\text {opt }}^{(n)}$ are tangent to the boundary between separable and nonseparable states.

Another advantage of ${ }^{(S)} \mathcal{W}_{\text {opt }}^{(n)}$ is that one can rewrite the $\mathcal{W}_{R}^{(n)}$ in terms of positive operators $\sigma_{S}^{\prime},\left|\psi_{00 \ldots 0}\right\rangle\left\langle\psi_{00 \ldots 0}\right|$ and some optimal EWs, i.e.,

$$
\mathcal{W}_{R}^{(n)}=\sum_{S} a_{S}^{(S)} \mathcal{W}_{o p t}^{(n)}+d_{1} \omega_{2}\left|\psi_{00 \ldots 0}\right\rangle\left\langle\psi_{00 \ldots 0}\right|+\sum_{S}\left(a_{S}+a_{S}^{\prime}\right) \sigma_{S}^{\prime} \quad S \varsubsetneqq N^{\prime}
$$

Therefore for positive $\omega_{2}$, the REWs can be decomposed as

$$
\mathcal{W}_{R}^{(n)}=\sum_{S}{ }^{(S)} \mathcal{Q}^{T_{\left(N^{\prime} \backslash S\right)}}+\mathcal{P}
$$

where ${ }^{(S)} \mathcal{Q}:={ }^{(S)} \mathcal{W}_{\text {opt }}^{(n)^{T}}{ }_{\left(N^{\prime} \backslash S\right)}$ and $\mathcal{P}$ is positive operator and in this case $\mathcal{W}_{R}^{(n)}$ can not detect PPT entangled states( non-separable density matrices with positive partial transpose with respect to all particles).

\section{Some special cases of $\mathcal{W}_{R}^{(n)}$}

In this section we discuss some special cases of REWs to enlighten the subject. 


\subsection{Multi-qubit reduction type EWs}

It is important both theoretically and experimentally to study multi-qubit entanglement and to provide EWs to verify that in a given multi-qubit state, entanglement is really present. The Eq. (4.5) for system of n-qubits reduces to

$$
\mathcal{W}_{R}^{(n)}=\sum_{S \varsubsetneqq N^{\prime}} b_{S} \sigma_{S}+2 b_{2, \ldots, n}\left|\psi_{00 \ldots 0}\right\rangle\left\langle\psi_{00 \ldots 0}\right|
$$

As mentioned before, the dimension of qubit-systems does not allow presence of $\sigma_{S}^{\prime}$.

The number of $\mathrm{P}^{\prime} \mathrm{s}$ is $2^{n-1}-1$, whereas the number of apexes is $2^{n-1}$. Putting the coordinates of apexes in Eq.(4.10) again indicates that all $a_{S} \geq 0$. The feasible region is $2^{n-1}-$ simplex of dimension $2^{n-1}-1$ surrounded by hypersurfaces defined by (4.11) with all $P^{\prime \prime}$ s eliminated.

\subsubsection{Three-qubit $(\mathrm{n}=3)$}

The first nontrivial example of REWs for multi-qubit system is three qubit REW

$$
\mathcal{W}_{R}^{(3)}=a_{1} I_{8}+\left(a_{2}-a_{1}\right) \sigma_{2}+\left(a_{3}-a_{1}\right) \sigma_{3}+2\left(a_{2,3}+a_{1}-a_{2}-a_{3}\right)\left|\psi_{000}\right\rangle\left\langle\psi_{000}\right|
$$

with eigenvalues $a_{1}, a_{2}, a_{3} \geq 0, \omega_{1}=a_{2}+a_{3}-a_{1}$ and $\omega_{2}=2 a_{2,3}-\omega_{1}$. In order to obtain the feasible region, we need the coordinates of apexes which are

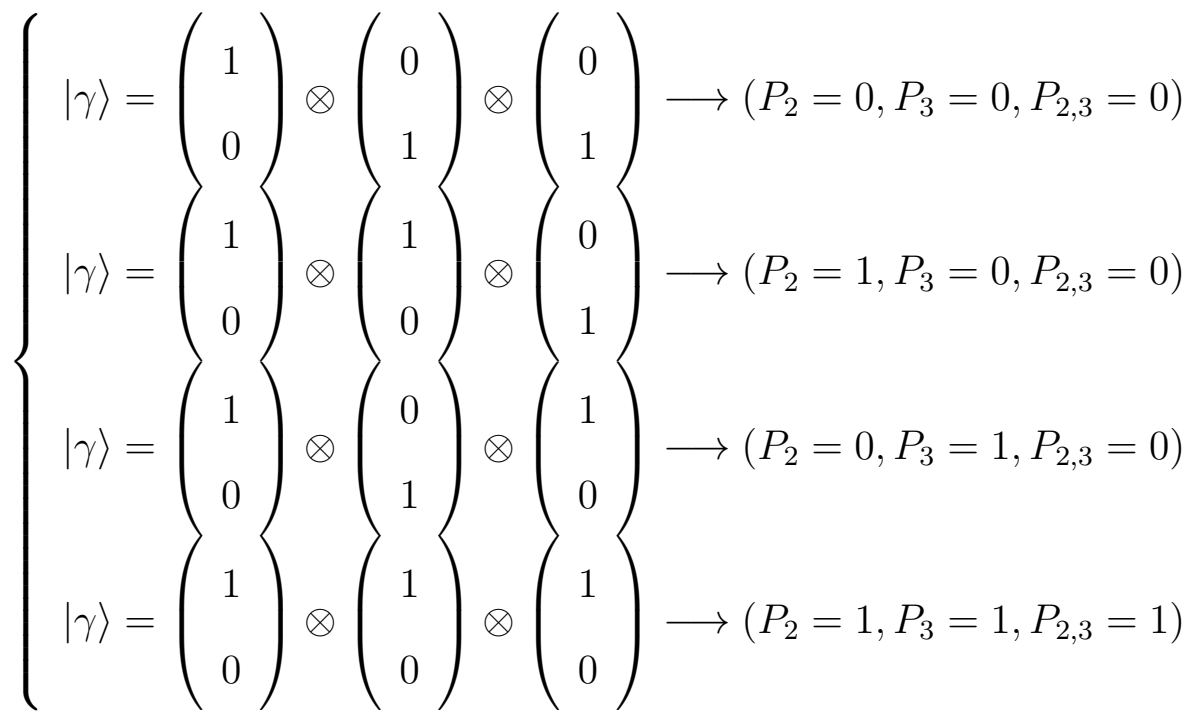


As we see the number of $P_{S}$ is three, while the number of optimal points is four. Therefore, there are four hyperplanes surrounding the feasible region, where the hyperplanes pass through each combination of three points out of four ones. (see Fig.1). Thus the problem can be reduced to

$$
\begin{aligned}
& \text { minimize } \quad \operatorname{Tr}\left(\mathcal{W}_{R}^{(3)}|\gamma\rangle\langle\gamma|\right) \\
& \text { subject to }\left\{\begin{array}{c}
1+P_{2,3}-P_{2}-P_{3} \geq 0, \\
P_{2}-P_{2,3} \geq 0, \\
P_{3}-P_{2,3} \geq 0, \\
P_{2,3} \geq 0
\end{array}\right.
\end{aligned}
$$

where the above given inequalities follow rather easily by taking the expectation value of the following optimal EWs

$$
\begin{gathered}
{ }^{2} \mathcal{W}_{\text {opt }}^{(3)}=a_{2}\left(\sigma_{2}-2\left|\psi_{000}\right\rangle\left\langle\psi_{\text {000 }}\right|\right) \\
{ }^{3} \mathcal{W}_{\text {opt }}^{(3)}=a_{3}\left(\sigma_{3}-2\left|\psi_{000}\right\rangle\left\langle\psi_{\text {000 }}\right|\right) \\
{ }^{1} \mathcal{W}_{\text {opt }}^{(3)}=a_{1}\left(I_{8}-\sigma_{2}-\sigma_{3}+2\left|\psi_{000}\right\rangle\left\langle\psi_{\text {ooo }}\right|\right)
\end{gathered}
$$

in pure product states, and as usual the optimal EWs can be written as partial transpose of the following positive operators

$$
\begin{aligned}
& { }^{1} \mathcal{W}_{\text {opt }}^{(3)}{ }^{T_{23}}=a_{1}(|100\rangle+|011\rangle)(\langle 100|+\langle 011|), \\
& { }^{2} \mathcal{W}_{\text {opt }}^{(3)^{T_{3}}}=a_{2}(|001\rangle+|110\rangle)(\langle 001|+\langle 110|), \\
& { }^{3} \mathcal{W}_{\text {opt }}^{(3)^{T_{2}}}=a_{3}(|010\rangle+|101\rangle)(\langle 010|+\langle 101|),
\end{aligned}
$$

respectively. Now the EW $\mathcal{W}_{R}^{(3)}$ can be written in terms of ${ }^{i} \mathcal{W}_{\text {opt }}$ as

$$
\begin{aligned}
\mathcal{W}_{R}^{(3)} & =a_{1}{ }^{1} \mathcal{W}_{\text {opt }}+a_{2}{ }^{2} \mathcal{W}_{\text {opt }}+a_{3}{ }^{3} \mathcal{W}_{\text {opt }}+2 \omega_{2}\left|\psi_{\text {ooo }}\right\rangle\left\langle\psi_{\text {ooo }}\right| \\
& =a_{1}{ }^{(1)} \mathcal{Q}^{T_{(23)}}+a_{2}{ }^{(2)} \mathcal{Q}^{T_{(3)}}+a_{3}{ }^{\left({ }^{3}\right)} \mathcal{Q}^{T_{(2)}}+2 \omega_{2} \mathcal{P}
\end{aligned}
$$

As mentioned above in section 4 , for $\omega_{2} \geq$ the $\mathrm{EW} \mathcal{W}_{R}^{(3)}$ becomes decomposable and can not detect PPT entangled states. 


\section{2 n-qudit $(d \otimes d \otimes \ldots \otimes d)$}

For $\mathrm{n}$ particles with the same dimensions the extra terms $\sigma_{S}^{\prime}$ will appear in EWs, provided that the requirement $|S| \leq n-3$ is met. Then the Eq.(4.5) becomes

$$
\mathcal{W}_{R}^{(n)}=\sum_{S \varsubsetneqq N^{\prime}} b_{S} \sigma_{S}+d b_{2, \ldots, n}\left|\psi_{00 \ldots 0}\right\rangle\left\langle\psi_{00 \ldots 0}\right|+\sum_{S \varsubsetneqq N^{\prime},|S| \leq n-3} a_{S}^{\prime} \sigma_{S}^{\prime}
$$

where

$$
\sigma_{S}^{\prime}=\sum_{i_{1}, \ldots, i_{n}=0}^{d-1}\left|i_{1}\right\rangle\left\langle i_{1}\right| \otimes O_{i_{2}}^{(2)} \otimes \ldots \otimes O_{i_{n}}^{\prime(n)}, \quad O_{i_{k}}^{(k)}= \begin{cases}\left|i_{1}\right\rangle\left\langle i_{1}\right| \delta_{i_{1} i_{k}} & k \in S \\ \left|i_{k}\right\rangle\left\langle i_{k}\right| \prod_{k \neq m=1}^{n}\left(1-\delta_{i_{k} i_{m}}\right) & k \notin S .\end{cases}
$$

We discuss below the most simple case: an REW consisting of just two qudits with the same dimension $d$, that is

$$
\mathcal{W}_{R}^{(2)}=a_{1} I_{d^{2}}+d\left(a_{2}-a_{1}\right)\left|\psi_{00}\right\rangle\left\langle\psi_{00}\right|
$$

Where for $a_{2}=0$ it reduces to the well known reduction EW ( the term "reduction-type EWs" for general $\mathcal{W}_{R}^{(n)}$ is inspired from this particular case). In this case we have only $P_{2}$ which can takes values between 0 and 1 . So the feasible region is just the line segment $0 \leq P_{2} \leq 1$. Putting 0 and 1 in $\mathcal{F}\left(P_{2}\right)=a_{1}+\left(a_{2}-a_{1}\right) P_{2}$ yields $a_{1} \geq 0$ and $a_{2} \geq 0$ respectively. The eigenvalues are $a_{1}$ and $2 a_{2}-a_{1}$ where the second one must be negative to ensure detecting some entangled states.

\subsection{Three particles $(2 \otimes 3 \otimes 4)$}

As a particular example of REWs with different dimension let us discuss three particles with $2 \otimes 3 \otimes 4$ dimensions

$$
\mathcal{W}_{R}^{(3)}=a_{1} I_{8}+\left(a_{2}-a_{1}\right) \sigma_{2}+\left(a_{3}-a_{1}\right) \sigma_{3}+2\left(a_{2,3}+a_{1}-a_{2}-a_{3}\right)\left|\psi_{000}\right\rangle\left\langle\psi_{000}\right|+a_{1}^{\prime} \sigma_{1}^{\prime}+a_{2}^{\prime} \sigma_{2}^{\prime}+a_{3}^{\prime} \sigma_{3}^{\prime}
$$

where we have

$$
\sigma_{1}^{\prime}=|012\rangle\langle 012|+| 013\rangle\langle 013|+| 021\rangle\langle 021|+| 022\rangle\langle 022|+| 023\rangle\langle 023|
$$




$$
\begin{gathered}
|102\rangle\langle 102|+| 103\rangle\langle 103|+| 120\rangle\langle 120|+| 122\rangle\langle 122|+| 123\rangle\langle 123| \\
\sigma_{2}^{\prime}=|002\rangle\langle 002|+| 003\rangle\langle 003|+| 112\rangle\langle 112|+| 113\rangle\langle 113| \\
\sigma_{3}^{\prime}=|020\rangle\langle 020|+| 121\rangle\langle 121|
\end{gathered}
$$

Here all possible $\sigma_{S}^{\prime}(S=\{2\},\{3\}$ and $\emptyset)$ can be appear. In this case feasible region lies in a space of dimension six. The coordinates of apexes and relevant pure product states $|\gamma\rangle$ are

$$
\begin{aligned}
& |\gamma\rangle=\left(\begin{array}{l}
1 \\
0
\end{array}\right) \otimes\left(\begin{array}{l}
0 \\
1 \\
0
\end{array}\right) \otimes\left(\begin{array}{l}
0 \\
1 \\
0 \\
0
\end{array}\right) \longrightarrow(0,0,0,0,0,0) \\
& |\gamma\rangle=\left(\begin{array}{l}
1 \\
0
\end{array}\right) \otimes\left(\begin{array}{l}
1 \\
0 \\
0
\end{array}\right) \otimes\left(\begin{array}{l}
0 \\
1 \\
0 \\
0
\end{array}\right) \longrightarrow(1,0,0,0,0,0) \\
& |\gamma\rangle=\left(\begin{array}{l}
1 \\
0
\end{array}\right) \otimes\left(\begin{array}{l}
0 \\
1 \\
0
\end{array}\right) \otimes\left(\begin{array}{l}
1 \\
0 \\
0 \\
0
\end{array}\right) \longrightarrow(0,1,0,0,0,0) \\
& |\gamma\rangle=\left(\begin{array}{l}
1 \\
0
\end{array}\right) \otimes\left(\begin{array}{l}
1 \\
0 \\
0
\end{array}\right) \otimes\left(\begin{array}{l}
1 \\
0 \\
0 \\
0
\end{array}\right) \longrightarrow(1,1,1,0,0,0) \\
& \longrightarrow\left(\begin{array}{l}
1 \\
0
\end{array}\right) \\
& \left.\qquad \begin{array}{l}
1 \\
0
\end{array}\right)
\end{aligned}
$$




$$
\begin{aligned}
& |\gamma\rangle=\left(\begin{array}{l}
1 \\
0
\end{array}\right) \otimes\left(\begin{array}{l}
0 \\
1 \\
0
\end{array}\right) \otimes\left(\begin{array}{l}
0 \\
0 \\
1 \\
0
\end{array}\right) \longrightarrow(0,0,0,1,0,0) \\
& |\gamma\rangle=\left(\begin{array}{l}
1 \\
0
\end{array}\right) \otimes\left(\begin{array}{l}
1 \\
0 \\
0
\end{array}\right) \otimes\left(\begin{array}{l}
0 \\
0 \\
1 \\
0
\end{array}\right) \longrightarrow(1,0,0,0,1,0) \\
& |\gamma\rangle=\left(\begin{array}{l}
1 \\
0
\end{array}\right) \otimes\left(\begin{array}{l}
0 \\
1 \\
0
\end{array}\right) \otimes\left(\begin{array}{l}
1 \\
0 \\
0 \\
0
\end{array}\right) \longrightarrow(0,1,0,0,0,1)
\end{aligned}
$$

Again choosing all combinations of six apexes out of seven ones, one can find the boundary of feasible region as

$$
\begin{gathered}
P_{1}^{\prime}, P_{2}^{\prime}, P_{3}^{\prime}, P_{2,3} \geq 0 \\
P_{2}-P_{2,3}-P_{2}^{\prime} \geq 0 \\
P_{3}-P_{2,3}-P_{3}^{\prime} \geq 0 \\
1+P_{2,3}-P_{2}-P_{3}-P_{1}^{\prime} \geq 0
\end{gathered}
$$

where the EWs corresponding to these hyperplanes are

$$
\begin{gathered}
{ }^{2} \mathcal{W}_{\text {opt }}=a_{2}\left(\sigma_{2}-2\left|\psi_{000}\right\rangle\left\langle\psi_{\text {ooo }}\right|-\sigma_{2}^{\prime}\right), \\
{ }^{3} \mathcal{W}_{\text {opt }}=a_{3}\left(\sigma_{3}-2\left|\psi_{000}\right\rangle\left\langle\psi_{\text {ooo }}\right|-\sigma_{3}^{\prime}\right), \\
{ }^{1} \mathcal{W}_{\text {opt }}=a_{1}\left(I_{24}-\sigma_{2}-\sigma_{3}+2\left|\psi_{000}\right\rangle\left\langle\psi_{\text {ooo }}\right|-\sigma_{1}^{\prime}\right) .
\end{gathered}
$$


Taking the partial transposition of ${ }^{i} \mathcal{W}_{\text {opt }}, i=1,2,3$ with respect to $\{2,3\} \backslash\{i\}$ yields

$$
\begin{aligned}
& { }^{1} \mathcal{W}_{\text {opt }}^{T_{23}}=a_{1}(|100\rangle+|011\rangle)(\langle 100|+\langle 011|), \\
& { }^{2} \mathcal{W}_{\text {opt }}^{T_{3}}=a_{2}(|001\rangle+|110\rangle)(\langle 001|+\langle 110|), \\
& { }^{3} \mathcal{W}_{\text {opt }}^{T_{2}}=a_{3}(|010\rangle+|101\rangle)(\langle 010|+\langle 101|),
\end{aligned}
$$

respectively. Evidently these EWs are optimal, since these have been written as the partial transposition of pure maximally entangled states.

\section{Bell-diagonal EWs by LP methods}

Recently multi-qubit Bell decomposable entangled witnesses (BDEWs) [14] have been introduced as

$$
\mathcal{W}_{B D}=\sum_{i_{1} i_{2} \ldots i_{n}=0,1} a_{i_{1} i_{2} \ldots i_{n}}\left|\psi_{i_{1} i_{2} \ldots i_{n}}\right\rangle\left\langle\psi_{i_{1} i_{2} \ldots i_{n}}\right|
$$

where $\left|\psi_{i_{1} i_{2} \ldots i_{n}}\right\rangle\left(d_{i}=2, i=1,2 \ldots, n\right)$ are n-qubit maximally entangled orthonormal states, i.e.,

$$
\left|\psi_{i_{1} i_{2} \ldots i_{n}}\right\rangle=\left(\sigma_{z}\right)^{i_{1}} \otimes\left(\sigma_{x}\right)^{i_{2}} \otimes \ldots \otimes\left(\sigma_{x}\right)^{i_{n}}\left|\psi_{00 \ldots 0}\right\rangle
$$

where $\sigma_{x}$ and $\sigma_{z}$ are usual Pauli matrices.

In general it is hard to find the BDEWs with feasible region of simplex type or even polygon type, namely those which can manipulated by LP method. Here we give two examples which are both set in LP problem, where only one of them (the first example) can be solved exactly by the prescription of this paper.

The first example is EW of the form

$$
\mathcal{W}_{1}=a I_{2^{n}}+2(b-a)\left|\psi_{00 \ldots 0}\right\rangle\left\langle\psi_{00 \ldots 0}|+2(c-a)| \psi_{00 \ldots 01}\right\rangle\left\langle\psi_{00 \ldots .01}\right|+(d-a) \sigma
$$

where

$$
\sigma=I_{2^{n}}-\left\{\left(\left|\psi_{011 \ldots 10}\right\rangle\left\langle\psi_{011 \ldots 110}|+| \psi_{11 \ldots 10}\right\rangle\left\langle\psi_{11 \ldots 10}\right|\right)+\left(\left|\psi_{011 \ldots 1}\right\rangle\left\langle\psi_{011 \ldots 1}|+| \psi_{11 \ldots 11}\right\rangle\left\langle\psi_{11 \ldots 11}\right|\right)\right.
$$




$$
\left.+\left(\left|\psi_{00 \ldots 0}\right\rangle\left\langle\psi_{00 \ldots 0}|+| \psi_{10 \ldots 0}\right\rangle\left\langle\psi_{10 \ldots .}\right|\right)+\left(\left|\psi_{00 \ldots 01}\right\rangle\left\langle\psi_{00 \ldots 01}|+| \psi_{10 \ldots 01}\right\rangle\left\langle\psi_{10 \ldots 01}\right|\right)\right\}
$$

The eigenvalues of $\mathcal{W}_{1}$ are $a, 2 b-a, 2 c-a, d$. This BDEW is similar to the one introduced in [14], where the extra term $\sigma$ is added to optimize the EWs corresponding to the boundary plane of feasible region. Suppose that $P_{00 \ldots 0}=2\left|\left\langle\psi_{00 \ldots .} \mid \gamma\right\rangle\right|^{2}, P_{00 \ldots 01}=2\left|\left\langle\psi_{00 \ldots 01} \mid \gamma\right\rangle\right|^{2}$ and $P=\operatorname{Tr}(\sigma|\gamma\rangle\langle\gamma|)$. Then the pure product states which produce the apexes are

$$
\left\{\begin{array}{l}
|\gamma\rangle=\left(\begin{array}{l}
1 \\
0
\end{array}\right) \otimes\left(\begin{array}{l}
0 \\
1
\end{array}\right) \otimes \ldots \otimes\left(\begin{array}{l}
0 \\
1
\end{array}\right) \longrightarrow\left(P_{00 \ldots 0}=0, P_{00 \ldots 01}=0, P=0\right) \\
|\gamma\rangle=\left(\begin{array}{l}
1 \\
0
\end{array}\right) \otimes\left(\begin{array}{l}
0 \\
1
\end{array}\right) \otimes\left(\begin{array}{l}
1 \\
0
\end{array}\right) \otimes \ldots \otimes\left(\begin{array}{l}
1 \\
0
\end{array}\right) \longrightarrow\left(P_{00 \ldots 0}=0, P_{00 \ldots 01}=0, P=1\right) \\
|\gamma\rangle=\left(\begin{array}{l}
1 \\
0
\end{array}\right) \otimes\left(\begin{array}{l}
1 \\
0
\end{array}\right) \otimes \ldots \otimes\left(\begin{array}{l}
1 \\
0 \\
1 \\
0 \\
0
\end{array}\right) \otimes \ldots\left(P_{00 \ldots 0}=1, P_{00 \ldots 01}=0, P=0\right) \\
|\gamma\rangle=\left(\begin{array}{l}
1 \\
0
\end{array}\right) \otimes\left(\begin{array}{l}
0 \\
1
\end{array}\right) \longrightarrow\left(P_{00 \ldots 0}=0, P_{00 \ldots 01}=1, P=0\right)
\end{array}\right.
$$

and consequently these yield the following hyperplanes surrounding the feasible region (see Fig.2)

$$
\begin{gathered}
P_{00 \ldots 0}, P_{00 \ldots 01}, P \geq 0 \\
1-P_{00 \ldots 0}-P_{00 \ldots 01}-P \geq 0
\end{gathered}
$$

The positivity of the last constraint come from the positivity of the expectation value of the following optimal EW

$$
\mathcal{W}_{\text {opt }}=I-2\left|\psi_{00 \ldots 0}\right\rangle\left\langle\psi_{00 \ldots 0}|-2| \psi_{00 \ldots 01}\right\rangle\left\langle\psi_{00 \ldots 01}\right|-\sigma
$$

in pure product states $|\gamma\rangle$, since it can be written as the partial transpose of a positive operator with respect to the fist particle as

$$
\mathcal{W}_{\text {opt }}^{T_{1}}=2\left(\left|\psi_{11 \ldots 10}\right\rangle\left\langle\psi_{11 \ldots 10}|+| \psi_{11 \ldots 11}\right\rangle\left\langle\psi_{11 \ldots 11}\right|\right)
$$


Now the remaining task is to solve the following LP problem

$$
\begin{array}{cc}
\text { minimize } & a+(b-a) P_{00 \ldots 0}+(c-a) P_{00 \ldots 01}+(d-a) P \\
\text { subject to } & 1-P_{00 \ldots 0}-P_{00 \ldots 01}-P \geq 0 \\
& P_{00 \ldots .}, P_{00 \ldots 01}, P \geq 0
\end{array}
$$

Thus, above problem is reduced to LP and can be solved by simplex method. Putting the apexes in Eq.(7.26) we deduce that $a, b, c, d$ should be positive. Now, the operator $\mathcal{W}_{1}$ fulfills the properties of EWs if at least one of its eigenvalues is negative, namely $2 b-a<0$ or $2 c-a<0$.

The second example which sets in LP problem is

$$
\mathcal{W}_{2}=a I_{2^{n}}+2^{n-1}(b-a)\left|\psi_{00 \ldots 0}\right\rangle\left\langle\psi_{00 \ldots 0}\left|+2^{n-1}(c-a)\right| \psi_{10 \ldots 0}\right\rangle\left\langle\psi_{10 \ldots 0}\right|
$$

which can not be solved by the prescription of this paper. Its feasible region can be determined by Lagrangian multiplier method, as it is discussed in [14]. Let $P_{00 \ldots 0}=2\left|\left\langle\psi_{00 \ldots 0} \mid \gamma\right\rangle\right|^{2}, P_{10 \ldots 0}=$ $2\left|\left\langle\psi_{10 \ldots 0} \mid \gamma\right\rangle\right|^{2}$. There the problem reduces to

$$
\begin{array}{r}
\text { minimize } \\
\text { subject to }\left\{\begin{array}{c}
a+2^{n-2}(b-a) P_{00 \ldots 0}+2^{n-2}(c-a) P_{10 \ldots 0} \\
\frac{1}{2^{n-2}}-P_{00 \ldots 0}+\left(1-\frac{1}{2^{n-2}}\right) P_{10 \ldots 0} \geq 0 \\
\frac{1}{2^{n-2}}-P_{10 \ldots 0}+\left(1-\frac{1}{2^{n-2}}\right) P_{00 \ldots 0} \geq 0 \\
P_{00 \ldots 0}, P_{10 \ldots 0} \geq 0
\end{array}\right.
\end{array}
$$

These constraints can not be reach by partial transposition approach, and the feasible region is estimated by convex hall of apexes (see Fig.3).

\section{Detecting some entangled states by $\mathcal{W}_{R}^{(n)}$}

This section is devoted to some entangled states which can be detected by general $\mathcal{W}_{R}^{(n)}$ and three-qubit REW $\mathcal{W}_{R}^{(3)}$. First we consider some Bell states. All of the Bell states $\left|\psi_{i_{0 \ldots 0} \ldots}\right\rangle$ can 
be detected by $\mathcal{W}_{R}^{(n)}$, since we have

$$
\begin{gathered}
\operatorname{Tr}\left(\mathcal{W}_{R}^{(n)}\left|\psi_{00 \ldots 0}\right\rangle\left\langle\psi_{00 \ldots 0}\right|\right)=\omega_{2}, \\
\operatorname{Tr}\left(\mathcal{W}_{R}^{(n)}\left|\psi_{i_{0} \ldots 0}\right\rangle\left\langle\psi_{i_{0} \ldots 0}\right|\right)=\omega_{1} \quad, \quad i \neq 0,
\end{gathered}
$$

therefore for $\omega_{2}<0$ one can detect $\left|\psi_{00 \ldots 0}\right\rangle$ and for $\omega_{1}<0$ the others modulated Bell states can be detected by $\mathcal{W}_{R}^{(n)}$. On the other hand, imposing some constraints on operator

$$
\rho_{i, 0, \ldots, 0}=\frac{1}{B \operatorname{Tr}\left(\rho_{s}\right)+D d_{1}}\left\{B\left(I-\sum_{j=0}^{d_{1}-1}\left|\psi_{j \quad \ldots 0}\right\rangle\left\langle\psi_{j \quad \ldots 0}\right|\right)+D d_{1}\left|\psi_{i \quad \ldots 0}\right\rangle\left\langle\psi_{i \quad 0 \ldots 0}\right|\right\},
$$

one can get a density matrix which can be detected by $\mathcal{W}_{R}^{(n)}$, where $\rho_{s}$ denotes the separable state inside the parenthesis on the righthand side. The positivity of $\rho_{i, 0, \ldots, 0}$ constrains $B$ and $D$ to be positive and in order to detect both $\rho_{0,0, \ldots, 0}$ and $\rho_{i, 0, \ldots, 0}, i \neq 0$, we must have

$$
\begin{aligned}
& B \operatorname{Tr}\left(\mathcal{W}_{R}^{(n)} \rho_{s}\right)+D d_{1} \omega_{2}<0 \\
& B \operatorname{Tr}\left(\mathcal{W}_{R}^{(n)} \rho_{s}\right)+D d_{1} \omega_{1}<0
\end{aligned}
$$

respectively. Because of the positivity of $D$, Eq.(8.30) is satisfied if $\omega_{2}<0$ and Eq.(8.31) is satisfied if $\omega_{1}<0$. Now, we can proceed our discussion further to detect PPT entangled states which is useful for determining non-decomposable region. Here we do not discuss the decomposability and non-decomposability issues in detail, since it needs the other opportunity and comes elsewhere. $\rho_{i, 0, \ldots, 0}$ is PPT states with respect to any subsystems of the particles, if $B-D$ be positive, so the Eq.(8.30) yields

$$
1 \leq \frac{B}{D}<\frac{-d_{1} \omega_{2}}{\operatorname{Tr}\left(\mathcal{W}_{R}^{(n)} \rho_{s}\right)} \quad \Longrightarrow \quad \omega_{2}<\varpi
$$

where

$$
\varpi:=-\frac{1}{d_{1}} \operatorname{Tr}\left(\mathcal{W}_{R}^{(n)} \rho_{s}\right)
$$

The above requirement makes $\rho_{0,0, \ldots, 0}$ a class of PPT entangled state which can be detected by $\mathcal{W}_{R}^{(n)}$. But to detect the other $\rho_{i, 0, \ldots, 0}$ we must have $\omega_{1}<\varpi$ which is impossible. This is in 
agreement with the discussion made in section 5. Now, combining thus obtained results with those of Eq. (5.12) which implies that $\mathcal{W}_{R}^{(n)}$ is decomposable provided that $\omega_{2}$ is positive, one can rather determine the decomposability and non-decomposability of REWs (see Fig.4).

Furthermore one can construct some entangled states which can be detected by particular REWs. As an example consider entangled density matrices

$$
\begin{aligned}
& \rho=\frac{1}{4 B+2 D}\left(B \sigma_{2}+2 D\left|\psi_{000}\right\rangle\left\langle\psi_{000}\right|\right) \\
& \rho^{\prime}=\frac{1}{4 B+2 D}\left(B \sigma_{3}+2 D\left|\psi_{000}\right\rangle\left\langle\psi_{000}\right|\right)
\end{aligned}
$$

which can be detected by three-qubit REW (6.15), with some constraints. The positivity of these states implies that $B, B+2 D \geq 0$ and the positivity of $\rho^{T_{3}}, \rho^{T_{2}}$ is achieved if $B \pm D \geq 0$. In order that, $\operatorname{Tr}\left(\mathcal{W}_{R}^{(3)} \rho\right)$ to be negative we should have $B\left(a_{2,3}+a_{2}\right)+D \omega_{2}<0$, where we have two possibilities: for $D>0$ we have $\omega_{2}<0$ and for $D<0$ we have $\omega_{2}>0$.

\section{$9 \quad$ Positive maps}

As it is shown in [23], there is a close connection between the positive maps and the entanglement witnesses, i.e., the Jamiołkowski isomorphism

$$
\begin{gathered}
d_{1} d_{2} \ldots d_{n}\left(I_{d_{1} \ldots d_{n}} \otimes \mathcal{E}\right)\left|\psi_{+}\right\rangle\left\langle\psi_{+}\right|=\mathcal{W}_{d_{1}, d_{1}^{\prime}, \ldots, d_{n}, d_{n}^{\prime}}^{(1,2, \ldots, 2 n)}, \quad d_{i} \leq d_{i}^{\prime} \quad, \quad i=1, \ldots, n \\
\mathcal{E}(\rho)=\operatorname{Tr}_{(1,3, \ldots, 2 n-1)}\left[\mathcal{W}_{d_{1}, d_{1}^{\prime}, \ldots, d_{n}, d_{n}^{\prime}}^{(1,2, \ldots, 2 n)}\left(\rho^{T} \otimes I_{d_{1}^{\prime} d_{2}^{\prime} \ldots d_{n}^{\prime}}\right)\right]
\end{gathered}
$$

where

$$
\left|\psi_{+}\right\rangle=\frac{1}{\sqrt{d_{1} d_{2} \ldots d_{n}}} \sum_{i_{1}=0}^{d_{1}-1} \sum_{i_{2}=0}^{d_{2}-1} \ldots \sum_{i_{n}=0}^{d_{n}-1}\left|i_{1}^{(1)} i_{1}^{(2)}\right\rangle\left|i_{2}^{(3)} i_{2}^{(4)}\right\rangle \ldots\left|i_{n}^{(2 n-1)} i_{n}^{(2 n)}\right\rangle
$$

is the maximally entangled state in $\mathcal{H}_{d_{1}}^{(1)} \otimes \mathcal{H}_{d_{1}^{\prime}}^{(2)} \otimes \ldots \otimes \mathcal{H}_{d_{n}}^{(2 n-1)} \otimes \mathcal{H}_{d_{n}^{\prime}}^{(2 n)}$. Hence the Jamiołkowski isomorphism is a one-to-one mapping between the set of trace preserving quantum operations

$$
\mathcal{E}: \mathcal{H}_{d_{1}}^{(1)} \otimes \mathcal{H}_{d_{2}}^{(3)} \otimes \ldots \otimes \mathcal{H}_{d_{n_{1}}}^{(2 n-1)} \longrightarrow \mathcal{H}_{d_{1}^{\prime}}^{(2)} \otimes \mathcal{H}_{d_{2}^{\prime}}^{(4)} \otimes \ldots \otimes \mathcal{H}_{d_{n_{1}}^{\prime}}^{(2 n)}
$$


and $d_{1} \times d^{\prime} \times \ldots \times d_{n} \times d_{n}^{\prime}$ EWs if $d_{i} \leq d_{i}^{\prime}$ for $i=1,2, \ldots, n$.

Now, using the Jamiołkowski isomorphism (9.35), we try to construct the positive maps connected with REWs. Evidently tensor product of some EWs is also an EW in higher dimension. To be more precise let $\mathcal{W}_{d_{1} d_{1}^{\prime} \ldots d_{n} d_{n}^{\prime}}^{(1,2, \ldots, 2 n)}$ be an EW acting on $\mathcal{H}_{d_{1}}^{(1)} \otimes \mathcal{H}_{d_{1}^{\prime}}^{(2)} \otimes \ldots \otimes \mathcal{H}_{d_{n}}^{(2 n-1)} \otimes \mathcal{H}_{d_{n}^{\prime}}^{(2 n)}$ then depending on possible partition

$$
n=n_{1}+n_{2}+\ldots+n_{m}, \quad n_{i} \geq 1
$$

one can construct an EW by tensor product of REWs as

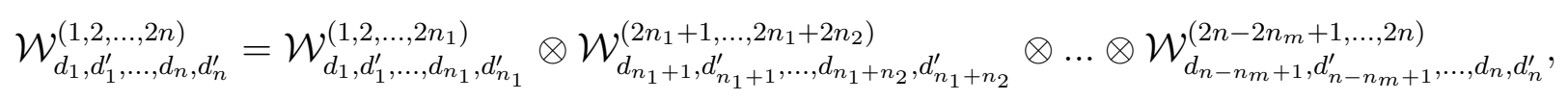

then using Jamiołkowski isomorphism (9.35), one can obtain the corresponding positive map. For instance, considering the tensor product of $n$ REWs(corresponding to the partition $n=$ $1+1+\ldots+1)$

$$
\mathcal{W}_{d_{1}, d_{1}^{\prime}, \ldots, d_{n}, d_{n}^{\prime}}^{(1,2, \ldots, 2 n)}=\bigotimes_{k=1}^{n} \mathcal{W}_{d_{k}, d_{k}^{\prime}}^{(2 k-1,2 k)}
$$

with

$$
\mathcal{W}_{d_{k}, d_{k}^{\prime \prime}}^{(2 k-1,2 k)}=a_{1}^{(2 k-1,2 k)} I+\left(a_{2}^{(2 k-1,2 k)}-a_{1}^{(2 k-1,2 k)}\right)\left|\psi_{00}^{(2 k-1,2 k)}\right\rangle\left\langle\psi_{00}^{(2 k-1,2 k)}\right|+a_{1}^{\prime(2 k-1,2 k)} \sigma_{1}^{(2 k-1,2 k)}
$$

acting on Hilbert space $\mathcal{H}_{d_{k}}^{(2 k-1)} \otimes \mathcal{H}_{d_{k}^{\prime}}^{(2 k)}$ with $\sigma_{1}^{\prime(2 k-1,2 k)}$ given in Eq. (4.7), we get the following positive map

$$
\mathcal{E}^{(n)}(\rho)=\sum_{S \subseteq\{1,3, \ldots, 2 n-1\}} \Gamma_{S} O_{S}
$$

where

$$
\begin{gathered}
\Gamma_{S}=\prod_{j \in N \backslash S}\left(a_{2}^{(2 j-1,2 j)}-a_{1}^{(2 j-1,2 j)}\right) \\
O_{S}=\bigotimes_{j_{i} \in S}\left(a_{1}^{\left(2 j_{i}-1,2 j_{i}\right)} I_{d_{j_{i}}}^{\left(j_{i}+1\right)}+a_{1}^{\left(2 j_{i}-1,2 j_{i}\right)} \sum_{k=d_{j_{i}}^{\prime}-1}^{d_{j_{i}}^{\prime}}|k\rangle\langle k|\right) \otimes T r_{j_{1} \ldots j_{|S|}}(\rho)
\end{gathered}
$$


and $\left(j_{1} \ldots j_{|S|}\right)$ is the ordered of $S$. Choosing all $a_{2}=a_{1}^{\prime}=0$ and $a_{1}=1$ yields the generalized reduction map introduced in [21]. As an example for $n=2$ one can easily verify that

$$
\begin{gathered}
\mathcal{E}^{(2)}(\rho)=\operatorname{Tr}_{1,3}\left[\mathcal{W}_{d_{1}, d_{1}, d_{2}, d_{2}}^{(1,2,3,4)}\left(\rho^{T_{1,3}} \otimes I_{d_{1} d_{2}}^{(2,4)}\right)\right]=a_{1}^{(1,2)} a_{1}^{(3,4)} \operatorname{Tr}(\rho) I_{d_{1}}^{(2)} \otimes I_{d_{2}}^{(4)}+a_{1}^{(1,2)}\left(a_{2}^{(3,4)}-a_{1}^{(3,4)}\right) I_{d_{1}}^{(2)} \otimes \operatorname{Tr}_{1}(\rho) \\
+a_{1}^{(3,4)}\left(a_{2}^{(1,2)}-a_{1}^{(1,2)}\right) \operatorname{Tr}_{3}(\rho) \otimes I_{d_{2}}^{(4)}+\left(a_{2}^{(1,2)}-a_{1}^{(1,2)}\right)\left(a_{2}^{(3,4)}-a_{1}^{(3,4)}\right) \rho \\
a_{1}^{(1,2)} a_{1}^{\prime(3,4)} \operatorname{Tr}(\rho) I_{d_{1}}^{(2)} \otimes \sum_{k=d_{2}}^{d_{2}^{\prime}-1}|k\rangle\left\langle k\left|+\left(a_{2}^{(1,2)}-a_{1}^{(1,2)}\right) a_{1}^{\prime(3,4)} \operatorname{Tr}_{3}(\rho) \otimes \sum_{k=d_{2}}^{d_{2}^{\prime}-1}\right| k\right\rangle\langle k| \\
+a_{1}^{\prime(1,2)}\left(a_{2}^{(3,4)}-a_{1}^{(3,4)}\right) \sum_{k=d_{1}}^{d_{1}^{\prime}-1}|k\rangle\left\langle k\left|\otimes \operatorname{Tr}_{1}(\rho)+a_{1}^{\prime(1,2)} a_{1}^{(3,4)} \operatorname{Tr}(\rho) \sum_{k=d_{1}}^{d_{1}^{\prime}-1}\right| k\right\rangle\langle k| \otimes I_{d_{2}}^{(4)} \\
+a_{1}^{\prime(1,2)} a_{1}^{\prime(3,4)} \operatorname{Tr}(\rho) \sum_{k=d_{1}}^{d_{1}^{\prime}-1}|k\rangle\left\langle k\left|\otimes \sum_{k=d_{2}}^{d_{2}^{\prime}-1}\right| k\right\rangle\langle k|
\end{gathered}
$$

Taking $a_{2}^{(1,2)}=a_{2}^{(3,4)}=a_{1}^{\prime(1,2)}=a_{1}^{\prime(3,4)}=0$ and $a_{1}^{(1,2)}=a_{1}^{(3,4)}=1$ yields

$$
\mathcal{E}^{(2)}(\rho)=\operatorname{Tr}(\rho) I_{d_{1}}^{(2)} \otimes I_{d_{2}}^{(4)}-I_{d_{1}}^{(2)} \otimes \operatorname{Tr}_{1}(\rho)-\operatorname{Tr}_{3}(\rho) \otimes I_{d_{2}}^{(4)}+\rho
$$

and choosing $a_{2}^{(1,2)}=a_{2}^{(3,4)}=0$ and $a_{1}^{(1,2)}=a_{1}^{(3,4)}=-a_{1}^{\prime(1,2)}=-a_{1}^{(3,4)}=1$ we reach the new reduction positive map for different dimensions

$$
\begin{gathered}
\mathcal{E}^{(2)}(\rho)=\operatorname{Tr}(\rho) I_{d_{1}}^{(2)} \otimes I_{d_{2}}^{(4)}-I_{d_{1}}^{(2)} \otimes \operatorname{Tr}_{1}(\rho)-\operatorname{Tr}_{3}(\rho) \otimes I_{d_{2}}^{(4)}+\rho-\operatorname{Tr}(\rho) I_{d_{1}}^{(2)} \otimes \sum_{k=d_{2}}^{d_{2}^{\prime}-1}|k\rangle\left\langle k\left|+\operatorname{Tr}_{3}(\rho) \otimes \sum_{k=d_{2}}^{d_{2}^{\prime}-1}\right| k\right\rangle\langle k| \\
+\sum_{k=d_{1}}^{d_{1}^{\prime}-1}|k\rangle\left\langle k\left|\otimes \operatorname{Tr}_{1}(\rho)-\operatorname{Tr}(\rho) \sum_{k=d_{1}}^{d_{1}^{\prime}-1}\right| k\right\rangle\left\langle k\left|\otimes I_{d_{2}}^{(4)}+\operatorname{Tr}(\rho) \sum_{k=d_{1}}^{d_{1}^{\prime}-1}\right| k\right\rangle\left\langle k\left|\otimes \sum_{k=d_{2}}^{d_{2}^{\prime}-1}\right| k\right\rangle\langle k|
\end{gathered}
$$

These examples show that one can construct more positive maps by making tensor product of EWs $\mathcal{W}_{R}^{(n)}$ in arbitrary way provided that the dimensionality condition $d_{i} \leq d_{i}^{\prime}$ is satisfied.

\section{Conclusion}

The generalized reduction type entanglement witnesses with simplex feasible regions are introduced, where the EWs corresponding to hyperplanes surrounding the feasible regions are 
optimal. These REWs are of types that their manipulation is reduced to LP problem and can be solved exactly by using the simplex method. As it shown above, the REWs are decomposable in cases if their second eigenvalue, namely $\omega_{2}$ becomes positive while for negative values of $\omega_{2}$, the decomposability or non-decomposability of REWs is still open for debate. Also various other interesting issues remain unsolved, such as keeping the REWs in realm of LP problems despite of adding some other operators or entangled states to them.

\section{Appendix I:}

Proof of the inequalities: $0 \leq P_{S}, P_{S}^{\prime}, P_{2, \ldots, n} \leq 1$.

In this appendix we prove that all $P_{S}, P_{S}^{\prime}$ and $P_{2, \ldots, n}$ take the values between 0 and 1 . The inequalities $0 \leq P_{S}, P_{S}^{\prime} \leq 1$ can be easily concluded from the following ones

$$
\begin{aligned}
& 0 \leq \operatorname{Tr}\left(\sigma_{S}|\gamma\rangle\langle\gamma|\right) \leq \prod_{k=1}^{n} \sum_{i=0}^{d_{k}-1}\left|\alpha_{i}^{(k)}\right|^{2}=1, \\
& 0 \leq \operatorname{Tr}\left(\sigma_{S}^{\prime}|\gamma\rangle\langle\gamma|\right) \leq \prod_{k=1}^{n} \sum_{i=0}^{d_{k}-1}\left|\alpha_{i}^{(k)}\right|^{2}=1 .
\end{aligned}
$$

For $P_{2, \ldots, n}$, the Cauchy-Schwartz inequality implies that

$$
P_{2, \ldots, n}:=d_{1}\left|\left\langle\psi_{00 \ldots 0} \mid \gamma\right\rangle\right|^{2}=\left|\sum_{i=0}^{d_{1}-1} \alpha_{i}^{(1)} \alpha_{i}^{(2)} \ldots \alpha_{i}^{(n)}\right|^{2}=\left|\left\langle\alpha^{(1)} \mid \beta\right\rangle\right|^{2} \leq \|\left|\alpha^{(1)}\right\rangle\left\|^{2}\right\||\beta\rangle \|^{2},
$$

where

$$
|\beta\rangle=\left(\begin{array}{c}
\alpha_{0}^{(2)} \alpha_{0}^{(3)} \ldots \alpha_{0}^{(n)} \\
\alpha_{1}^{(2)} \alpha_{1}^{(3)} \ldots \alpha_{1}^{(n)} \\
\vdots \\
\alpha_{d_{1}-1}^{(2)} \alpha_{d_{1}-1}^{(3)} \ldots \alpha_{d_{1}-1}^{(n)}
\end{array}\right),
$$

finally using the following inequality

$$
\||\beta\rangle \|^{2}=\sum_{i=0}^{d_{1}-1}\left|\alpha_{i}^{(2)} \alpha_{i}^{(3)} \ldots \alpha_{i}^{(n)}\right|^{2} \leq \prod_{k=2}^{n} \sum_{i=0}^{d_{k}-1}\left|\alpha_{i}^{(k)}\right|^{2}=1,
$$


one can conclude that $0 \leq P_{2, \ldots, n} \leq 1$

\section{Appendix II:}

Proof of the feasible region of (4.11).

In order to obtain the feasible region of (4.11), we need to evaluate the expectation value of optimal EWs

$$
{ }^{(S)} \mathcal{W}_{o p t}^{(n)}=a_{S}\left(\sigma_{S}+\sum_{S \subsetneq S^{\prime} \neq N^{\prime}}(-1)^{|S|+\left|S^{\prime}\right|} \sigma_{S^{\prime}}+d_{1}(-1)^{|S|+\left|N^{\prime}\right|}\left|\psi_{00 \ldots 0}\right\rangle\left\langle\psi_{00 \ldots 0}\right|-\sigma_{S}^{\prime}\right) \quad S \varsubsetneqq N^{\prime}
$$

in pure product states $|\gamma\rangle\langle\gamma|$ where ${ }^{(\emptyset)} \mathcal{W}_{\text {opt }}^{(n)}={ }^{(1)} \mathcal{W}_{\text {opt }}^{(n)}$. Now by taking the partial transpose of ${ }^{(S)} \mathcal{W}_{\text {opt }}^{(n)}$ with respect to $\left(N^{\prime} \backslash S\right)$ we have

$$
{ }^{(S)} \mathcal{W}_{\text {opt }}^{(n)^{T}}{ }^{\left.T^{\prime} \backslash S\right)}=a_{S} \sum_{i \neq j}^{d_{1}-1}\left|\Psi_{i j}^{(S)}\right\rangle\left\langle\Psi_{i j}^{(S)}\right|
$$

where

$$
\begin{gathered}
\left|\Psi_{i j}^{(S)}\right\rangle:=\left|\alpha_{i j}^{(1)}\right\rangle \otimes\left|\alpha_{i j}^{(2)}\right\rangle \otimes \ldots \otimes\left|\alpha_{i j}^{(n)}\right\rangle+\left|\beta_{i j}^{(1)}\right\rangle \otimes\left|\beta_{i j}^{(2)}\right\rangle \otimes \ldots \otimes\left|\beta_{i j}^{(n)}\right\rangle, \\
\left|\alpha_{i j}^{(k)}\right\rangle=\left\{\begin{array}{lll}
|i\rangle & \text { if } \quad k \in 1 \cup S \\
|j\rangle & \text { if } \quad k \notin 1 \cup S
\end{array} \quad, \quad\left|\beta_{i j}^{(k)}\right\rangle=\left\{\begin{array}{lll}
|j\rangle & \text { if } & k \in 1 \cup S \\
|i\rangle & \text { if } & k \notin 1 \cup S
\end{array}\right.\right.
\end{gathered}
$$

Noting that all of these operators are positive definite and using the relation

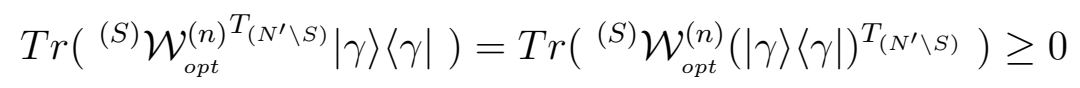

yields all feasible regions which are simplexes.

\section{Appendix III:}

Proof of the optimality of ${ }^{(S)} \mathcal{W}_{\text {opt }}^{(n)}$.

Here in this appendix we try to prove that witness ${ }^{(S)} \mathcal{W}_{\text {opt }}^{(n)}$ is optimal, to this aim we give the proof for the special case ${ }^{(2)} \mathcal{W}_{\text {opt }}^{(n)}$, since the proof of general case is rather similar to this 
particular one. According to the Reference [13], the $\mathrm{EW}^{(2)} \mathcal{W}_{o p t}^{(n)}$, is optimal if for all positive operator $\mathrm{P}$ and $\varepsilon>0$, the following new Hermitian operator

$$
\mathcal{W}_{\text {new }}=(1+\varepsilon)^{(2)} \mathcal{W}_{\text {opt }}^{(n)}-\varepsilon P
$$

is not anymore an EW. Suppose that there is a positive operator $P$ and $\epsilon \geq 0$ such that $\mathcal{W}_{\text {new }}={ }^{(2)} \mathcal{W}_{\text {opt }}^{(n)}-\epsilon P$ is yet an EW. Let the positive operator $P$ be the pure projection operator $\left|\psi_{i}\right\rangle\left\langle\psi_{i}\right|$, since an arbitrary positive operator can be written as sum of pure states with positive coefficients as $P=\sum_{i} \lambda_{i}\left|\psi_{i}\right\rangle\left\langle\psi_{i}\right|$.

Now, one should note that the expectation value of the operator ${ }^{(2)} \mathcal{W}_{\text {opt }}^{(n)}$ in pure product states $|\gamma\rangle$ will vanish if they satisfy the following equation

$$
A_{i} B_{j}^{*}+A_{j} B_{i}^{*}=0
$$

with

$$
A_{i}=\left(\alpha_{1}\right)_{i}\left(\alpha_{2}\right)_{i} \quad, \quad B_{j}=\left(\alpha_{3}\right)_{j}\left(\alpha_{4}\right)_{j} \ldots\left(\alpha_{n}\right)_{j}
$$

But, it is straightforward to see that, for $A_{i}, B_{j} \in \mathbb{R}$, the pure state $|\psi\rangle\langle\psi|$ will be similar to one of the $\left|\Psi_{i j}^{(2)}\right\rangle\left\langle\Psi_{i j}^{(2)}\right|$ with $i \neq j$ with

$$
\left|\Psi_{i j}^{(2)}\right\rangle:=|i\rangle \otimes|i\rangle \otimes|j\rangle \otimes \ldots \otimes|j\rangle+|j\rangle \otimes|j\rangle \otimes|i\rangle \otimes \ldots \otimes|i\rangle \quad i, j=0, \ldots, d_{1}-1
$$

concluding that an arbitrary $P$ has the form: $P=\sum_{i \neq j}^{d_{1}-1} a_{i j}\left|\Psi_{i j}^{(2)}\right\rangle\left\langle\Psi_{i j}^{(2)}\right|$ with $a_{i j} \geq 0$. Finally, substituting Eq. (I-6]) in the following expression

$$
\operatorname{Tr}(P|\gamma\rangle\langle\gamma|)=\sum_{i j} a_{i j}\left|A_{i} B_{j}+A_{j} B_{i}\right|^{2}=\sum_{i j} a_{i j}\left|\frac{A_{j}}{B_{j}}\right|^{2}\left|B_{j}^{*} B_{i}-B_{j} B_{i}^{*}\right|^{2}=0
$$

and choosing $B_{i}$ 's such that $B_{j}^{*} B_{i} \neq B_{j} B_{i}^{*}$ yields $a_{i j}=0$ and consequently one can conclude that $P=0$. 


\section{References}

[1] J. Preskill, The Theory of Quantum Information and Quantum Computation (California Inatitute of Technology, Pasadena, CA, 2000), http://www.theory.caltech.edu/poeole/preskill/ph229/.

[2] M. A. Nielsen and I. L. Chuang, Quantum Computation and Quantum Information (Cambridge University Press, Cambridge, 2000).

[3] The Physics of Quantum Information: Quantum Cryptography, Quantum Teleportation and Quantum Computation, edited by D. Bouwmeester, A. Ekert, and A. Zeilinger (Springer, New York, 2000).

[4] M. Horodecki, P. Horodecki and R. Horodecki, Springer Tracts Mod. Phys. 173, 151 (2001).

[5] M. Lewenstein, D. Bruß, J.I. Cirac, B. Kraus, M. Kus, J. Samsonowicz, A. Sanpera, and R. Tarrach, J. Mod. Opt. 47, 2841 (2000).

[6] B. M. Terhal, Theor. Comput. Sci. 287, 313 (2002) .

[7] D. Bruß, J. Math. Phys. 43, 4237 (2002).

[8] J. S. Bell, Physics (N.Y.) 1, 195 (1964).

[9] A. Peres, Phys. Rev. Lett. 77, 1413 (1996).

[10] M. Horodecki, P. Horodecki, and R. Horodecki, Phys. Lett. A 223, 1 (1996).

[11] C. H. Bennett, D. P. DiVincenzo, J. A. Smolin, and W. K. Wootters, Phys. Rev. A 54, 3824 (1996).

[12] W. K. Wootters, Phys. Rev. Lett. 80, 2245 (1998). 
[13] M. Lewenstein, B. Kraus, J.I. Cirac, and P. Horodecki, Phys. Rev. A 62, 052310 (2000); ibid. 63, 044304 (2001).

[14] M. A. Jafarizadeh, M. Rezaee, S. K. A. Seyed Yagoobi, Phys. Rev. A 72, 062106 (2005).

[15] M. A. Jafarizadeh, M. Rezaee, S. Ahadpour, Phys. Rev. A 74, 042335 (2006).

[16] E. Störmer, Acta Math. 110, 233 (1963); S. L. Woronowicz, Rep. Math. Phys. 10, 165 (1976); M. D. Choi, Proc. Sympos. Pure Math. 38, 583 (1982).

[17] A. C. Doherty, P. A. Parrilo, and F. M. Spedalieri, Phys. Rev. Lett. 88, 187904 (2002).

[18] A. C. Doherty, P. A. Parrilo, and F. M. Spedalieri, Phys. Rev. A. 69, 022308 (2004).

[19] R. O. Vianna, A. C. Doherty, eprint quant-ph/0608095 (2006).

[20] S. Boyd and L. Vandenberghe, Convex Optimization, Cambridge University Press, (2004).

[21] W. Hall, Phys. Rev. A 72, 022311 (2005)

[22] A. Acín, D. Bruß, M. Lewenstein, and A. Sanpera, Phys. Rev. Lett. 87, 040401 (2001).

[23] A. Jamiołkowski, Rep. Math. Phys. 3, 275 (1972). 


\section{Figure Captions}

Figure-1: 3-simplex displaying the feasible region of three-qubit REW.

Figure-2: 3-simplex displaying the feasible region of multi-qubit BDEW $\mathcal{W}_{1}$.

Figure-3: Convex polygon displaying the boundaries of the feasible region for multi-qubit $\operatorname{BDEW~} \mathcal{W}_{2}$.

Figure-4: Decomposable and non-decomposable regions of REWs: for $\omega_{2} \geq 0$ the REWs are decomposable, for $\omega_{2}<\varpi$ the REWs are non-decomposable and in dashed region, the decomposability or non-decomposability of REWs is still open for debate. 


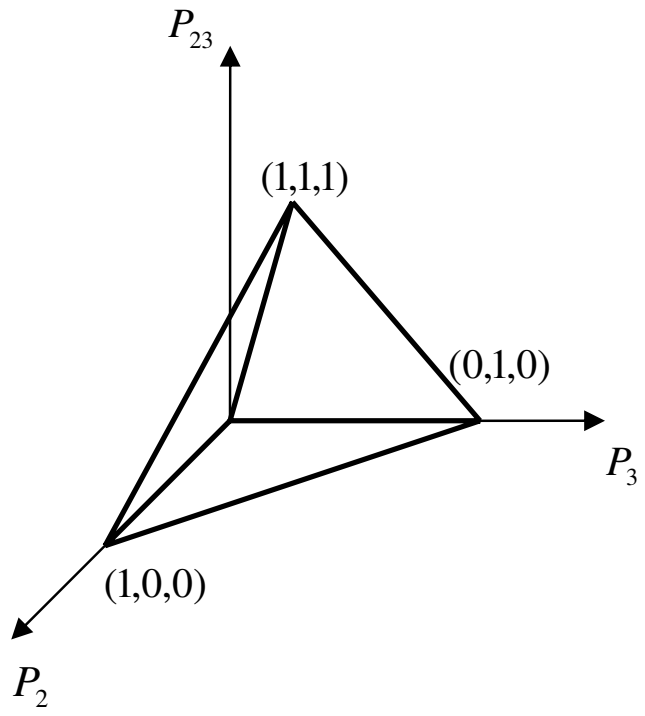

Fig. (1) 


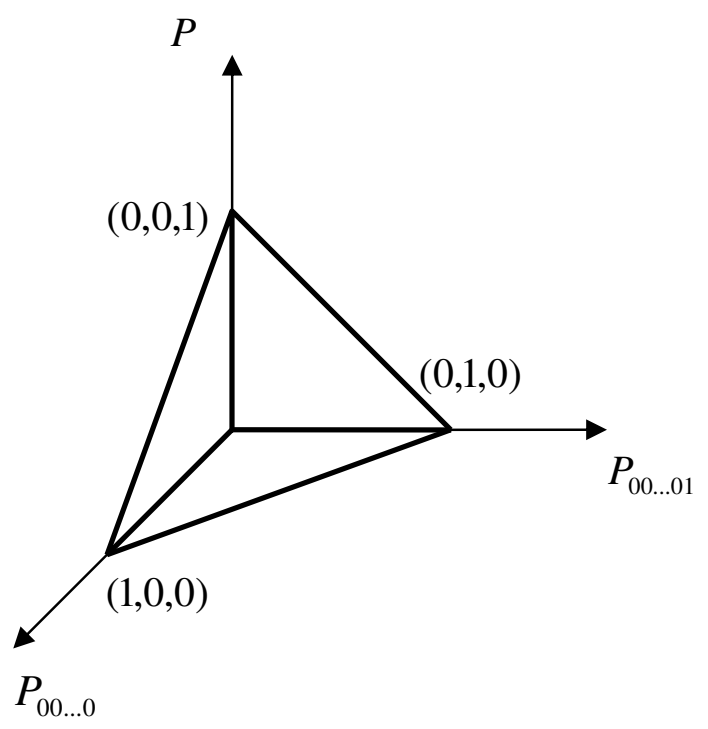

Fig. (2) 


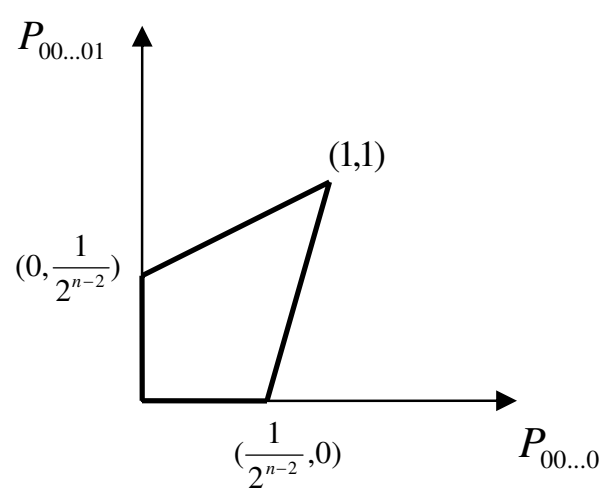

Fig. (3) 


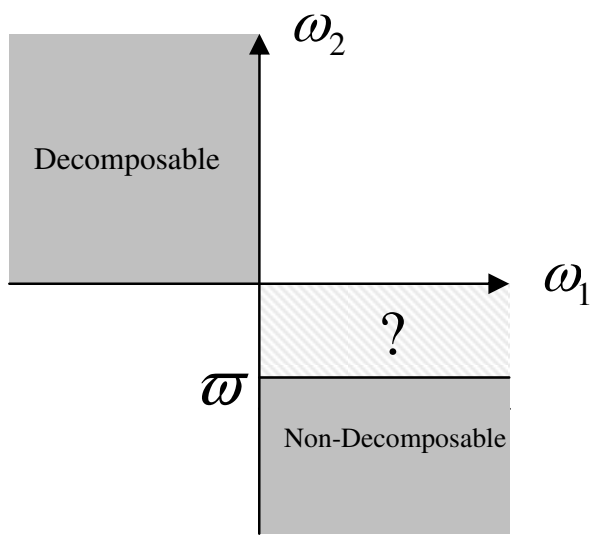

Fig. 4 